\title{
A STUDY OF THE PERFORMANCE OF THE MSR VEGETATION INDEX, USING PROBABILISTIC AND GEOSTATISTICAL METHODS
}

\author{
Skianis G. Aim. ${ }^{1}$, Vaiopoulos D. ${ }^{1}$, and Nikolakopoulos K. ${ }^{2}$ \\ ${ }^{1}$ National and Kapodistrian University of Athens, Faculty of Geology and Geoenvironment, \\ RemoteSensingLaboratory,skianis@geol.uoa.gr,vaiopoulos@geol.uoa.gr \\ ${ }^{2}$ I.G.M.E., Mesogion Str. 70, 115 27, Athens
}

\begin{abstract}
In the present paper is studied the effect of the MSR (Modified Soil Ratio) vegetation index on multispectral digital images, with the aid of probability theory and geostatistics. Using proper distributions to describe the histograms of the image at the red and infrared band zones, an analytical expression of the distribution $g$ of the MSR values is deduced. The study of the behaviour of $g$ shows that the ratio of the standard deviation to the mean value of the MSR image is higher than that of the NDVI vegetation index, which is quite often used. This means that the MSR vegetation index produces images with a good contrast. It was also observed that the MSR image has a better signal to noise ratio than that of the NDVI image. Finally, the autocorrelograms of the MSR and NDVI images showed that the tonality differences between adjacent pixels of the MSR image are slightly stronger than those of the NDVI image. The general conclusion is that the MSR vegetation index produces images with a good contrast and a high signal to noise ratio, which could aid in making a reliable mapping of the vegetation cover of the area under study.
\end{abstract}

Key words: distribution, histogram, signal to noise ratio, autocorrelogram, NDVI.

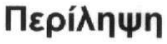

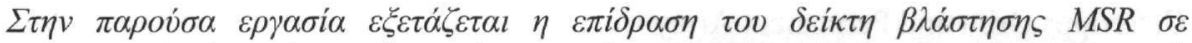

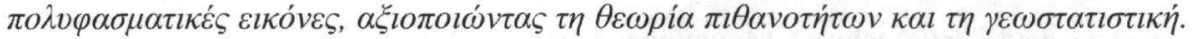

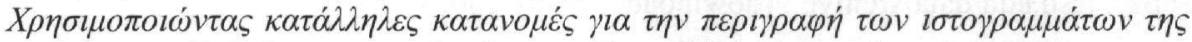

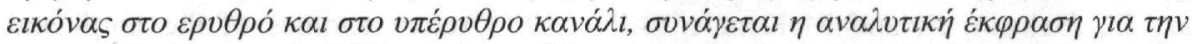

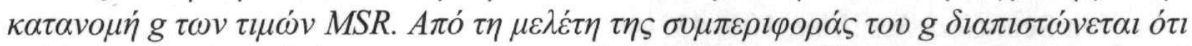

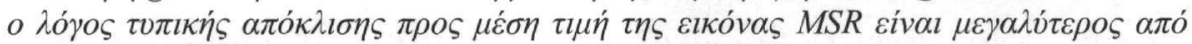

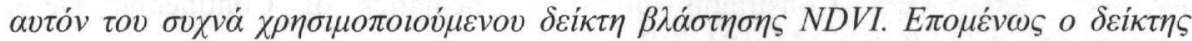

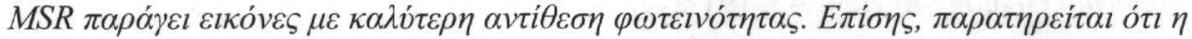

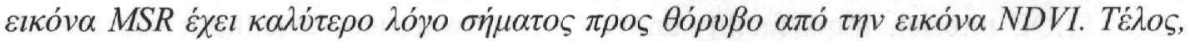

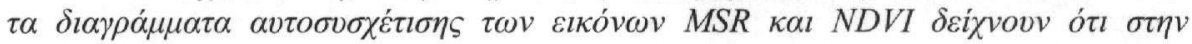

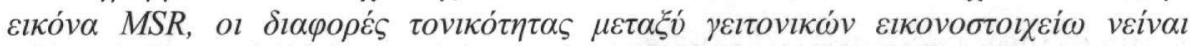
$\varepsilon \lambda \alpha \varphi \rho \alpha \dot{~ \varepsilon v \tau o v o ́ t \varepsilon \rho \varepsilon \varsigma ~ \alpha \pi o ́ ~ o ́ \sigma o ~ \sigma \tau \eta v ~ \varepsilon l \kappa o ́ v \alpha ~ N D V I . ~ T o ~ \gamma \varepsilon v \imath к o ́ ~ \sigma o \mu \pi \varepsilon ́ \rho \alpha \sigma \mu \alpha ~ \varepsilon i ́ v \alpha l ~ o ́ \tau l ~ o ~}$

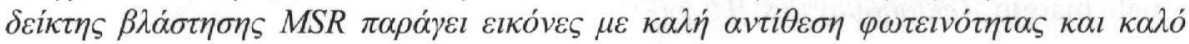




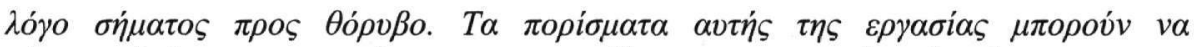

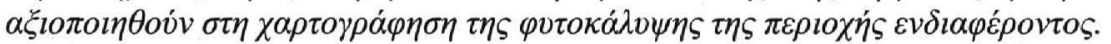

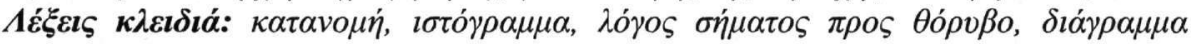

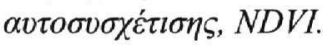

\section{Introduction}

In geological and environmental research with remote sensing technology, spectral band ratios are often used as vegetation indices, for mapping the vegetation cover of the area under study. Various vegetation indices have been introduced, mainly based on empirical criteria of response over vegetation types, soils or geological targets of interest (Jensen 1996, Rouse et al. 1973, Deering et al. 1975, Huete 1988, Faust 1989, Baret et al. 1989, Baret and Guyot 1991, Qi et al. 1994, Burgan 1996, Rondeaux et al. 1996, Coulibaly and Goita 2006). A great effort has been done in extracting information about vegetation cover parameters such as the Leaf Area Index (LAI) or the forest biomass, from vegetation indices and various reflectance bands (Chen \& Cihlar 1996, Fassnacht et al. 1997, Spanner et al. 1990, Brown et al. 2000, Chen 1996, Nemani et al. 1993, Fernandes et al. 2004, Rahman et al. 2005). In these approaches, remote sensing data are compared with ground data and correlation coefficients are computed. In certain cases, the sensitivity of various vegetation indices is assessed with the aid of mathematical models which associate the vegetation cover with its reflectance at various bands (Goel 1988, 1989, Verhoef 1998, Haboudane et al. 2004).

Recently, we have developed an alternative methodology of assessing the efficiency of vegetation indices with the aid of probability theory, which has already been applied for the study of the NDVI and the Simple Vegetation Index SVI (Vaiopoulos et al. 2004), for the Transformed Vegetation Index TVI (Skianis et al. 2004a) and for the Soil Adjusted Vegetation Index SAVI (Skianis et al. 2004b). The methodological difference between this recently developed probabilistic approach and other efforts is that the former is centered on the statistical behavior of the vegetation index under study, using theorems about bivariate distributions and appropriate distributions which may help in describing the histograms of the frequency bands. This means that the whole approach is focused on the mathematical structure of the function which defines the vegetation index and not on its functionality at different ground types or its correlation with biophysical parameters.

In the present paper, the probabilistic approach, combined with geostatistical (spatial autocorrelation) analysis is applied on the problem of the performance of the Modified Soil Ratio MSR. This vegetation index has been introduced by Chen (1996), in order to suppress the effects of the variable soil reflectance underneath the canopy.

The MSR vegetation index is defined by (Chen 1996):

\section{Equation 1-Definition of the MSR}

$$
M S R=u=\frac{r-1}{\sqrt{r}+1}
$$

$r$ is the ratio of the Near Infrared (NIR) to Red brightness value (or reflectance). Therefore the mathematical expression for the simple ratio $r$ is given by:

\section{Equation 2}

$r=\frac{N I R}{\operatorname{Red}}$ 
The MSR vegetation index has been used to estimate the LAI of the area under study (Fernandes et al. 2004, Haboudane et al. 2004). It's algebraic structure presents a certain similarity with the Normalized Differences Vegetation Index NDVI, which is quite often used in mapping the vegetation cover and is defined by (Liang 2004):

\section{Equation 3-Definition of the NDVI}

$$
N D V I=u=\frac{r-1}{r+1}
$$

In figure 1 are presented the variations of MSR and NDVI values against $r$. It can be observed that the MSR values are considerably bigger than the NDVI values, for $r$ more than 1.5 . These differences may influence the statistical parameters and the qualitative characteristics of the images of the vegetation indices.

The subject of this paper is the statistical performance of the MSR vegetation index, compared to that of the NDVI, which is quite often employed and may be considered as a standard reference vegetation index. The whole study is centered on the behavior of the histogram of the MSR image, the image signal to noise ratio and the spatial autocorrelation of the brightness values of the pixels. The results and conclusions of this paper may be useful in mapping the land cover of an area under study, with the aid of multispectral images and remote sensing technology.

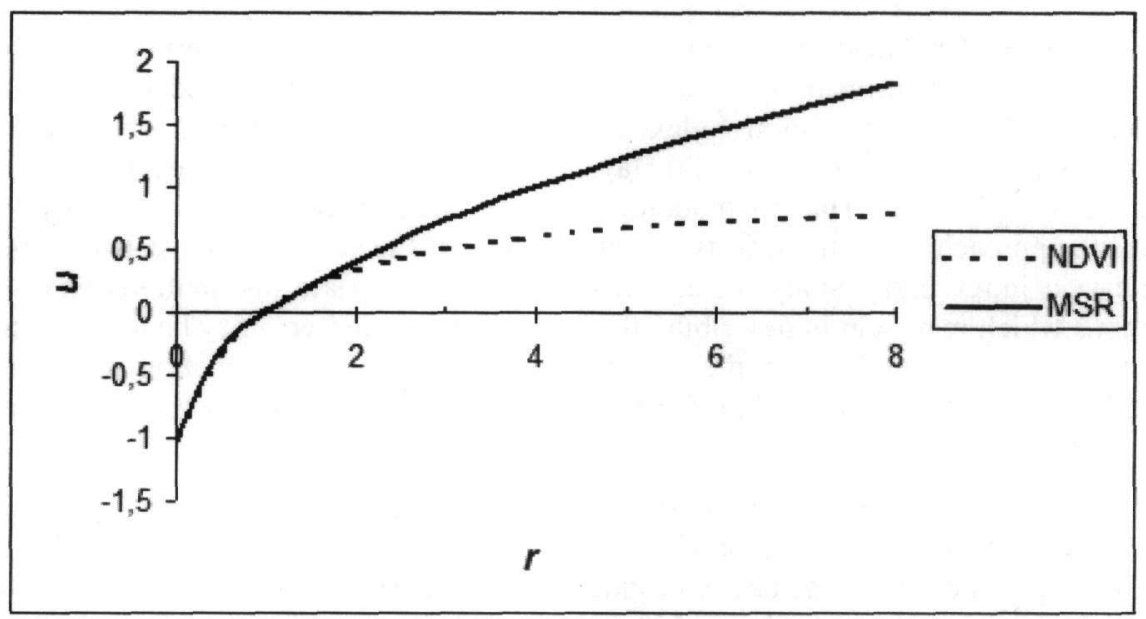

Figure 1 - MSR and NDVI values against $r$

\section{The histogram of the MSR image}

An image of a vegetation index with a broad histogram has a good contrast, which may help in detecting targets with a different tonality. A measure of the width of the histogram can be the standard deviation $\sigma$ or the ratio $\sigma / \mu$. A narrow vegetation index histogram (with a small standard deviation) may be broadened by histogram stretching. However two pixels with the same initial tonality can not have a varied tonality in the histogram stretched image, even if they represent different land cover types. On the other hand, if further processing of the vegetation index images (monitoring temporal changes in land cover, for example) is supposed to take place, histogram stretching should be avoided since it distorts the original values of the pixels. Therefore a good criterion for the efficiency of a vegetation index may be the standard deviation or the ratio $\sigma / \mu$ of the image of the vegetation index.

Vaiopoulos et al. (2004) have shown that using proper distributions, it is possible to find an analytical expression for the distribution $f(r)$ of the values of the simple ratio $r$, which is given by: 


\section{Equation 4-distribution of $\boldsymbol{r}$ values}

$$
f(r)=\frac{2 \lambda r}{\left(\lambda r^{2}+1\right)^{2}}
$$

The quantity $\lambda$ depends on the standard deviations of the histograms of the Near Infrared and Red channels, stdev(NIR) and stdev(Red) respectively, and may be defined by (Vaiopoulos et al. 2004):

\section{Equation 5}

$\lambda=\left[\frac{\operatorname{stdev}(\operatorname{Re} d)}{\operatorname{stdev}(N I R)}\right]^{2}$

The value $u$ of the MSR vegetation index is a function of the simple ratio $r$, as it can be seen in equation 1. According to Spiegel (1977), the distribution $g(u)$ of the MSR values can be found by the distribution $f(r)$ of the $r$ values, by the following relation:

\section{Equation 6}

$$
g(u)=f[r(u)] \cdot\left|\frac{d r}{d u}\right|
$$

Combining equation 4 with equation 6 gives:

\section{Equation 7-distribution of the MSR values}

$$
g(u)=\frac{4 \lambda(u+1)^{3}}{\left[\lambda(u+1)^{4}+1\right]^{2}}
$$

On the other hand, the distribution $g(u)$ of the NDVI values is given by (Vaiopoulos et.al. 2004):

\section{Equation 8-distribution of the NDVI values}

$$
g(u)=\frac{4 \lambda\left(1-u^{2}\right)}{\left[\lambda(1+u)^{2}+(1-u)^{2}\right]^{2}}
$$

In figure 2 are shown the distributions of the MSR and NDVI values, according to equations 7 and 8 , respectively. These distributions represent the histograms of the MSR and NDVI images, which are produced by applying these two vegetation indices on a multispectral image. It can be observed that the distribution of the MSR values is right skewed (positive skewness), due to the fact that the MSR takes values that can be quite higher than unity (the NDVI values can not be more than unity). Generally, the positive skewness of the histogram of the MSR image is clearly expressed when the standard deviation of the brightness values of the Red channel does not exceed the standard deviation of the NIR channel. The NDVI histogram has a negative skewness when the standard deviation of the Red channel is less than that of the NIR channel.

A quantitative criterion of the efficiency of the MSR vegetation index, compared to that of the NDVI, can be the ratio standard deviation/mean value of the image of the vegetation index. A high ratio expresses a wide range of values around the mean value, which indicates an image with a good contrast, where different land cover types can be clearly discriminated. A small ratio standard deviation/mean value expresses a narrow range of values around the mean value, which indicates an image with a bad contrast and poor discrimination between different land cover types. 


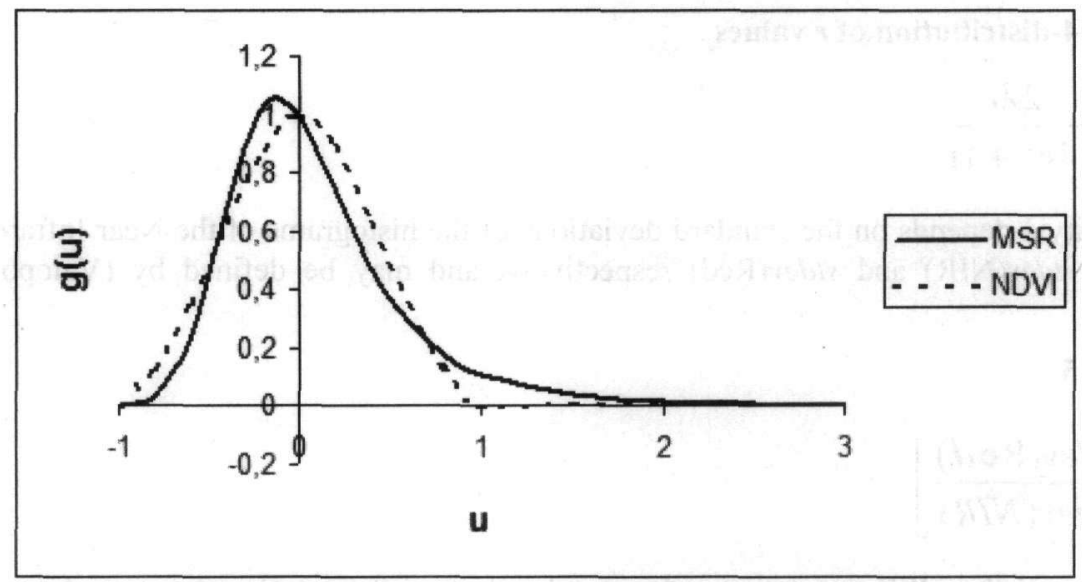

Figure 2 - Distributions of the values of the MSR and NDVI vegetation indices. $\lambda=1$

The standard deviation $\sigma$ can be computed according to the following relation:

\section{Equation 9-standard deviation}

$$
\sigma=\sqrt{\int_{R}(u-\mu)^{2} g(u) d u}
$$

$R$ is the integration range. For the MSR vegetation index, the integration range is from -1 to $\infty$. For the NDVI, the integration range is from -1 to +1 .

$\mu$ is the mean value of the distribution $g(u)$, and it is given by:

\section{Equation 10-mean value}

$$
\mu=\int_{R} u g(u) d u
$$

The mean values of the NDVI are quite close to zero, especially when the standard deviations of the Infrared and Red band zones, do not differ considerably. For this reason, in order to avoid very high ratios $\sigma / \mu$, the $u$ values of the NDVI and the MSR vegetation indices, were rescaled to the ranges $[0,2]$ and $[0, \infty)$, respectively. In such a rescaling process, the standard deviation $\sigma$ remains the same and the mean value $\mu$ is equal to the non scaled mean value plus unity.

The $\sigma / \mu$ values of the MSR vegetation index were computed numerically, according to the equations 7,9 and 10 , for various $\lambda$ values. The $\sigma / \mu$ values of the NDVI were also computed, according to the equations 8,9 and 10 . In figure 3 is shown the variation of $\sigma / \mu$ against $\lambda$, for an MSR and an NDVI image.

It can be observed that for the MSR vegetation index, the ratio $\sigma / \mu$ is practically constant for all $\lambda$ values. On the other hand, the $\sigma / \mu$ of the NDVI is smaller than the $\sigma / \mu$ of the MSR for relatively small $\lambda$ values. For $\lambda$ bigger than 6 , the $\sigma / \mu$ of the NDVI becomes bigger than that of the MSR. The physical meaning of the curves of figure 3 is that the MSR image is expected to have a better contrast than the NDVI image, if $\lambda$ is not very big.

The two vegetation indices were applied on a Landsat TM image of Zakynthos Island, which was taken in July 1989. In figures 4 and 5 are presented the MSR and the NDVI image, respectively. 


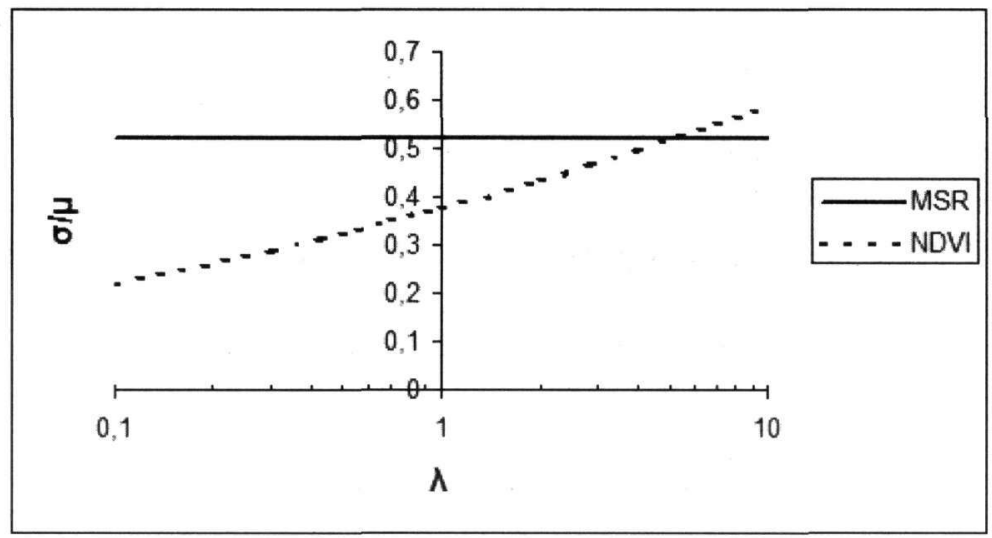

Figure 3 - Variation of $\sigma / \mu$ against $\lambda$

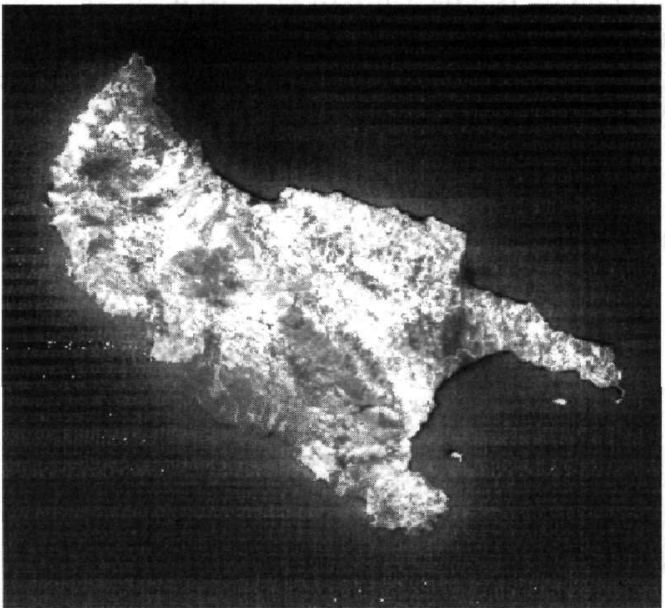

Figure 4 - The MSR image of Zakynthos Island

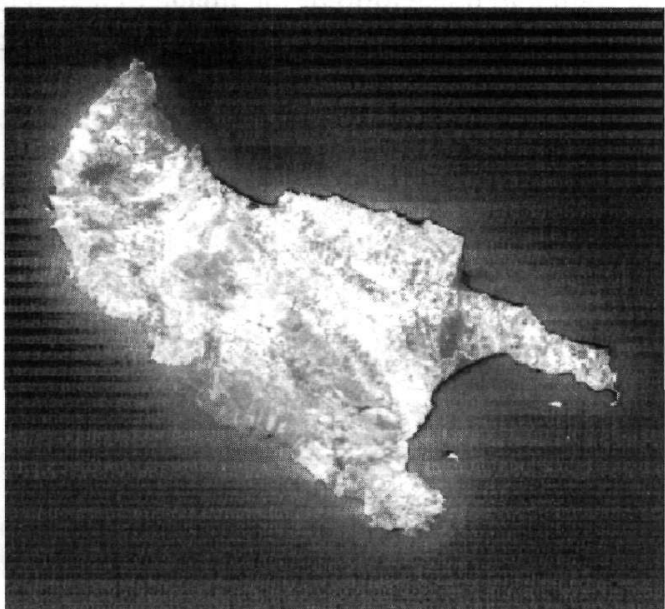

Figure 5 - The NDVI image of Zakynthos Island

It can be observed that the NDVI image has a diffuse brightness and a relatively weak contrast between the regions with poor vegetation (dark tonality) and the regions with a dense vegetation cover (bright tonality). The tonality contrast of the MSR image is stronger. On the other hand, some bright spots appear at the maritime region of the MSR image, which, obviously, don't have any physical meaning and they are probably due to very low brightness values at the pixels of the Landsat image.

The standard deviation of the Red channel is 16.038 and the standard deviation of the NIR channel is 34.458 . Therefore, according to equation 5 , the parameter $\lambda$ is equal to 0.217 .

In Table 1 are presented the theoretically predicted $\sigma / \mu$ values, according to the graphs of figure 3 , for $\lambda=0.217$. In the same table, the actual $\sigma / \mu$ values of the NDVI and MSR images are included. The quantity $\sigma / \mu$ of the NDVI image was calculated for a $[0,2]$ range of rescaled vegetation index values. The quantity $\sigma / \mu$ of the MSR image was calculated for a $[0, \infty)$ range of rescaled vegetation index values.

There is a very good agreement between the theoretically predicted and the actual $\sigma / \mu$ value for the MSR image, and a less good agreement between these two values for the NDVI image. It must be pointed out that the theoretical $\sigma / \mu$ values were calculated according to the distributions $g(u)$ of 
the equations 7 and 8, which are mere approximations of the histograms of the images of the vegetation indices. Very good agreement between theoretical predictions and actual values, is generally not expected. The most important is that theory and real data agree that, in qualitative terms, the $\sigma / \mu$ value of the MSR image is quite bigger than the $\sigma / \mu$ value of the NDVI image, which means that the MSR image has a better contrast.

\section{Table 1 - Theoretically predicted and actual statistical parameters}

\begin{tabular}{|l|l|l|}
\hline Image & $\sigma / \mu$ theoretical & $\sigma / \mu$ actual \\
\hline NDVI & 0.265 & 0.182 \\
\hline MSR & 0.523 & 0.525 \\
\hline
\end{tabular}

\section{The signal to noise ratio of the MSR image}

The signal to noise ratio of the image of a vegetation index is a measure of how well the vegetation index responds over regions with a different vegetation cover, in the presence of signals which are irrelevant to the targets of interest (noise). In this paper, the Near Infrared and Red brightness values are considered to contain a Gaussian noise with a constant standard deviation $\sigma_{\mathrm{n}}$.

The signal to noise ratio SNR is defined by (Schowengerdt 1997):

\section{Equation 11-definition of the signal to noise ratio}

$$
S N R=\frac{\sigma}{\sigma_{u}}
$$

$\sigma_{u}$ is the standard deviation of a $u$ value of the vegetation index (MSR or NDVI) at a certain pixel. $\sigma_{u}$ generally varies from pixel to pixel, since it depends on the NIR and Red brightness values of each pixel. $\sigma$ is the standard deviation of the tonality distribution $g(u)$ and it is defined according to equation 9. The distribution $g(u)$ is given by equation 7 (for the MSR) and by equation 8 (for the NDVI).

$\sigma_{u}$ is given by (Vaiopoulos et al. 2004):

\section{Equation 12}

$$
\sigma_{u}=\sigma_{n} \sqrt{\left(\frac{\partial u}{\partial(N I R)}\right)^{2}+\left(\frac{\partial u}{\partial(\operatorname{Re} d)}\right)^{2}}
$$

Combining the equations $1,2,7,9,10,11$ and 12 gives, after certain algebraic manipulation, the following expression for the signal to noise ratio of an image of the MSR vegetation index, SNR(MSR):

\section{Equation 13-the signal to noise ratio of an MSR image}

$$
\operatorname{SNR}(M S R)=\frac{2 \cdot \operatorname{Re} d \cdot \sigma(M S R)}{\sigma_{n}} \sqrt{\frac{r^{2}+1}{r}}
$$

$\sigma$ (MSR) is the standard deviation of the MSR image, which depends on the parameter $\lambda . r$ is the ratio of the pixel brightness value at the Near Infrared band to that of the Red band, according to the equation 2. 
Combining the equations $2,3,8,9,10,11$ and 12 gives the following expression for the signal to noise ratio of an image of the NDVI vegetation index:

\section{Equation 14-the signal to noise ratio of an NDVI image}

$S N R(N D V I)=\frac{\operatorname{Re} d \cdot \sigma(N D V I)}{2 \sigma_{n}} \cdot \frac{\sqrt{r^{2}+1}}{(r+1)^{2}}$

$\sigma(\mathrm{NDVI})$ is the standard deviation of the NDVI image, which depends on the parameter $\lambda$.

Combining the equations 13 and 14, gives:

\section{Equation 15-the ratio SNR(MSR) to SNR(NDVI)}

$$
\frac{S N R(M S R)}{S N R(N D V I)}=\frac{4 \sigma(M S R)}{\sigma(N D V I)} \cdot \frac{(r+1)^{2}}{r^{2}+1} \cdot \sqrt{r}
$$

In figure 6 is presented the variation of the ratio SNR(MSR)/SNR(NDVI) against $r$, according to equation 15. It can be observed that SNR(MSR)/SNR(NDVI) is almost always more than unity and it increases with $r$. The ratio SNR(MSR)/SNR(NDVI) can be less than unity for very small values of $r$. For example, for $\lambda=0.217$ (which is the $\lambda$ value of the Landsat image), the value of $r$ beyond which this ratio exceeds unidy, is $r_{0}=0.0094$. Integrating equation 4 for $\lambda=0.217$ and with integration range $[0.0094, \infty]$, the percentage of the pixels with $\mathrm{SNR}(\mathrm{MSR}) / \mathrm{SNR}(\mathrm{NDVI})>1$ is found to be $99.998 \%$. The general conclusion of the mathematical analysis of the signal to noise ratio of the MSR and NDVI images is that the signal to noise ratio of the former is better (higher) than that of the latter.

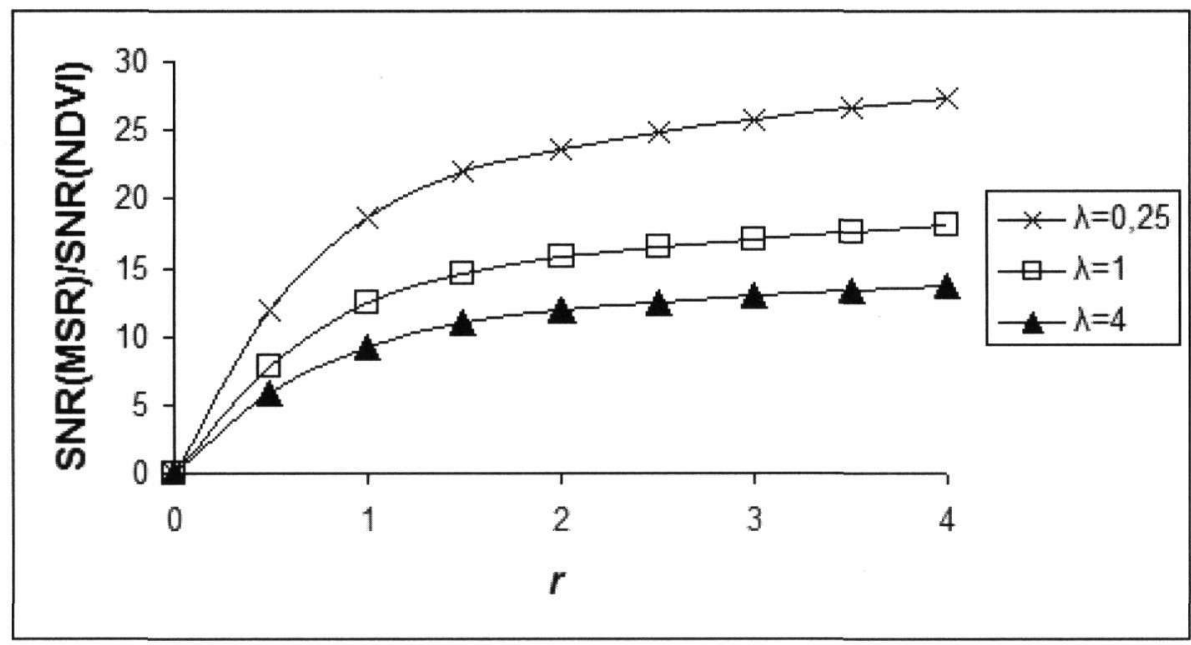

Figure 6 - SNR(MSR)/SNR(NDVI) variation against $r$

In order to compare in practice the signal to noise ratio of the MSR image with that of the NDVI, the signal to noise ratio for every pixel of the MSR and the NDVI images of the Landsat scene of Zakynthos Island was computed, using a proper software which was developed in the ERDAS Imagine environment. Then, for each pixel, the ratio SNR(MSR)/SNR(NDVI) was found.

In figure 7 is presented the histogram of the SNR(MSR)/SNR(NDVI) values. 


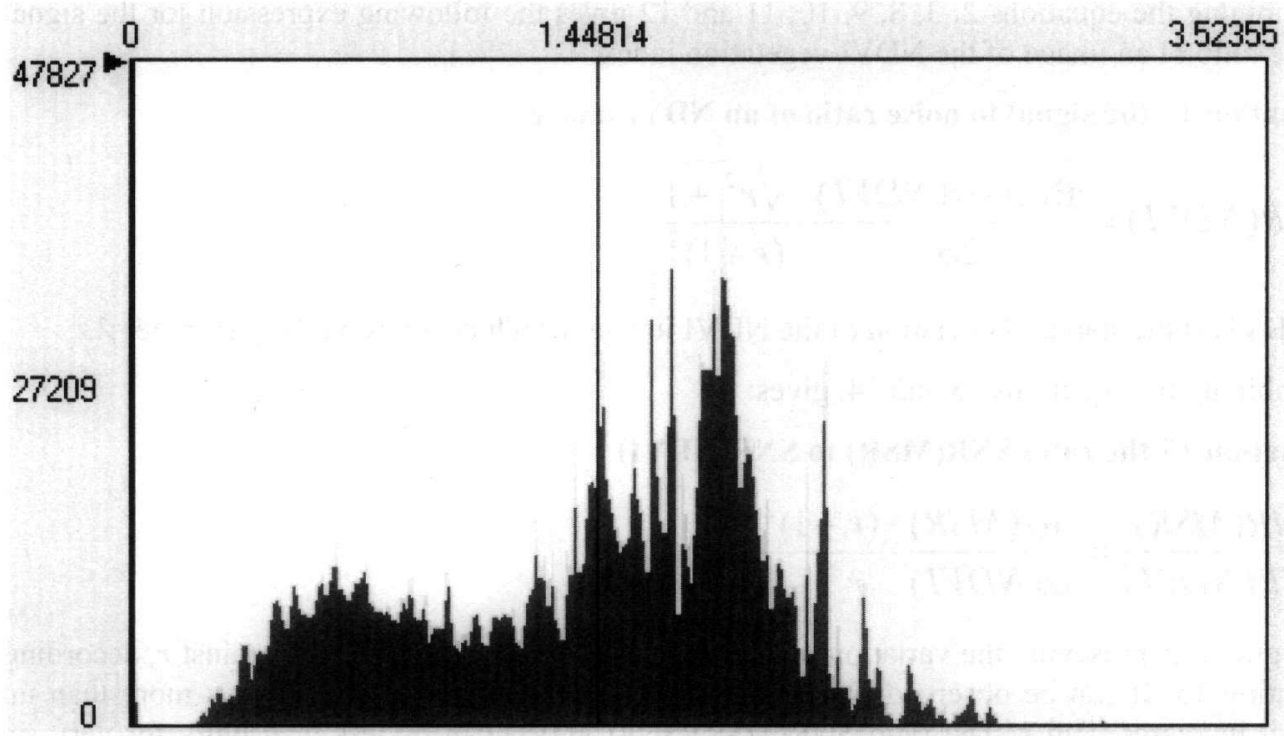

Figure 7 - Histogram of the SNR(MSR)/SNR(NDVI) values (product of the Landsat image)

The values of the ratio SNR(MSR)/SNR(NDVI) range from zero to 3.52. The mean value is 1.45. A further analysis of the histogram showed that for the $75 \%$ of the pixels of the Landsat image, the ratio is more than unity. In other words, most of the pixels have a better (higher) signal to noise ratio for the MSR vegetation index than for the NDVI vegetation index. This means that, in quantitative terms, there is a considerable deviation between the theoretical prediction and the experimentation on the Landsat image, since the theoretically estimated percent of pixels with SNR(MSR)/SNR(NDVI) $>1$ is almost $100 \%$. In qualitative terms however, theory and experimental data show that, for most pixels, the signal to noise ratio of the MSR image is expected to be better than that of the NDVI image.

\section{The spatial autocorrelation of the MSR image}

The spatial autocorrelation of a digital image is a measure of the spatial variation of the tonality. A high spatial autocorrelation between two pixels at distance $d$ means that the spatial variation of the tonality from zero to $d$ presents a smooth tendency and the tonality of a certain pixel presents some relevance with the tonality of the other pixels at distance $d$. A small spatial autocorrelation at distance $d$ means that the tonality from zero to $d$ varies rapidly and the tonality of a certain pixel is irrelevant the tonality of the other pixels at distance $d$.

The autocorrelograms of the MSR and NDVI images were computed, using ILWIS software package, in order to form a picture of the spatial variation of these two vegetation indices, in quantitative terms. In figure 7 are presented the autocorrelograms of the MSR and the NDVI image, in the horizontal direction. In the vertical direction, the autocorrelograms are essentially the same, which means that there is no anisotropy on the spatial patterns of the images.

It can be observed that the autocorrelogram of the MSR image almost coincides with that of the NDVI image. A more careful examination of the two autocorrelograms, shows that the autocorrelation of the MSR is a little bit smaller than that of the NDVI, for every distance $d$. This means that the spatial variation of the NDVI values is slightly smoother than that of the MSR values; in other words, the tonalities of pixels of a certain region of the MSR image are less grouped (more variable) compared to those of the NDVI image. 


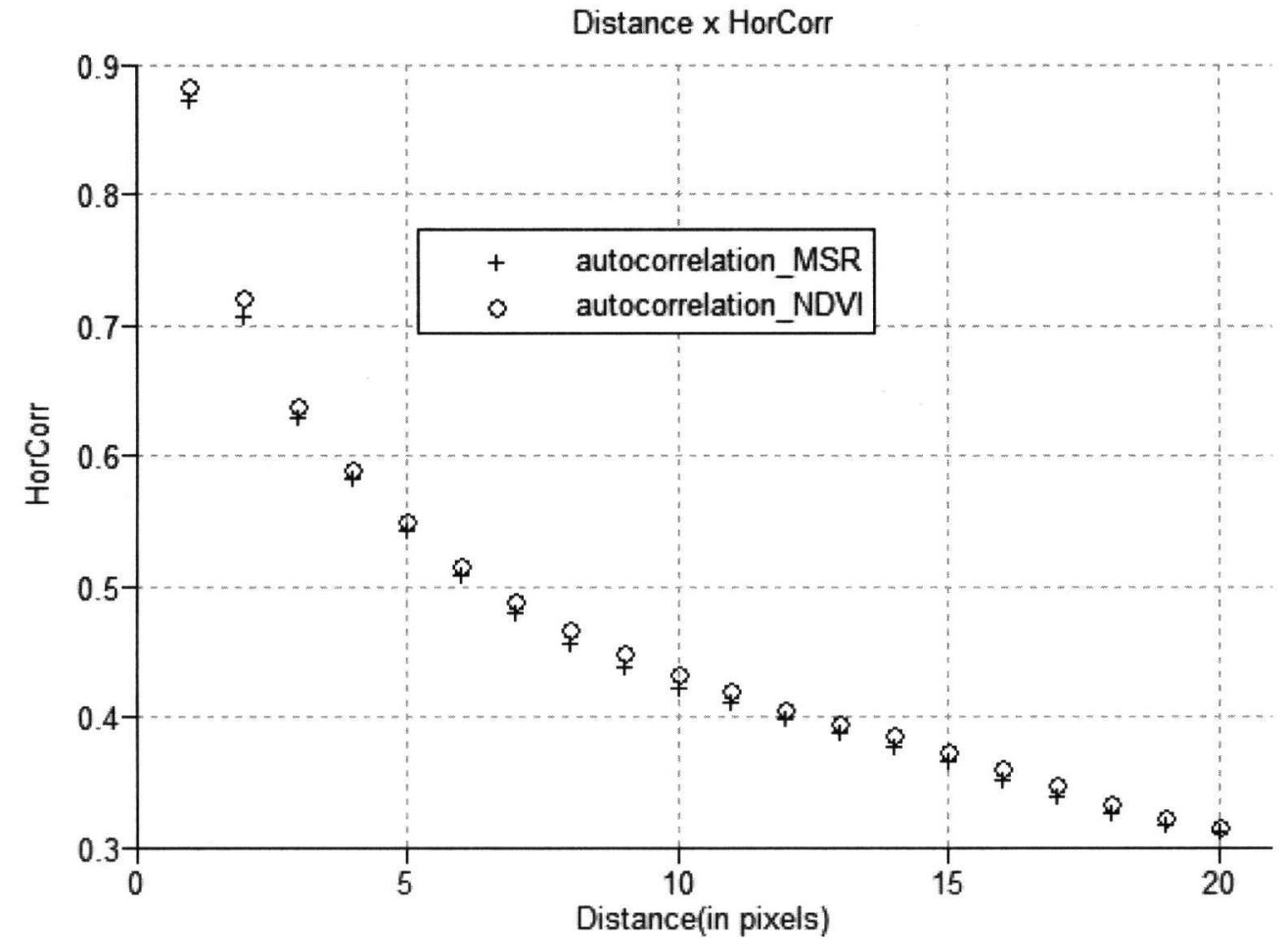

Figure 8 - The autocorrelograms of the MSR and NDVI images

\section{Conclusions}

According to the mathematical analysis and the experimentation with the Landsat image, the following conclusions may be deduced:

The statistical behaviour of the MSR and NDVI images depends on the ratio of the standard deviations of the NIR and Red channels of the multispectral image. For equal, more or less, standard deviations of the two channels, the histogram of the MSR image is right skewed and that of the NDVI is symmetrical. Generally, the right skewness of the MSR image is clearly expressed when the standard deviation of the tonality of the Red Channel is not bigger than that of the NIR channel. Because of this right skewness, the MSR image does not present the diffuse brightness which may be observed in the NDVI image, when the NDVI histogram has a zero or negative skewness, as a result of a not bigger standard deviation of the Red channel than that of the NIR channel.

The standard deviation/mean value ratio of the MSR image, is generally bigger than that of the NDVI image. This means that the MSR image has a better contrast. The signal to noise ratio of the MSR image is also better than that of the NDVI image.

The autocorrelograms of the MSR and NDVI images of the Landsat multispectral scene of Zakynthos island, are not considerably different, but they showed that the tonalities of the pixels of the MSR image have a more intensive spatial variation than those of the NDVI image.

On the other hand, the MSR image may contain some noise (spots with high brightness), which does not appear in the NDVI image. 
The general conclusion is that the MSR vegetation index produces images with a better tonality contrast and a better signal to noise ratio than the NDVI images, which are quite often used in environmental research.

The results and conclusions of this paper may be useful in mapping the land cover of an area under study, with the aid of multispectral images and remote sensing technology.

\section{References}

Baret, F., and Guyot, G., 1991. Potentials and limits of vegetation indices for LAI and APAR assessment, Remote Sensing of Environment 35, 161-173.

Baret, F., Guyot, G., and Major, D.J., 1989. TSAVI: a vegetation index which minimizes soil brightness effects on LAI and APAR estimation, Proc. IGARSS'89 and $12^{\text {th }}$ Canadian Symposium on Remote Sensing. Vancouver, Canada, 1355-1358.

Brown, L., Jin, M.C., Lablanc, S.G., and Cihlar, J., 2000. A shortwave infrared modification to the simple ratio for LAI retrieval in boreal forests: An image and model analysis, Remote Sensing of Environment, 71, 16-25.

Burgan, R.E., 1996. Use of Remotely Sensed Data for Fire Danger Estimation, Earsel Advances in Remote Sensing. Remote Sensing and GIS applications for Forest Fire Management, 4(4), $1-8$.

Chen, J., 1996. Evaluation of vegetation indices and modified simple ratio for boreal applications, Canadian. Journalof Remote Sensing, 22, 229-242.

Chen, J., and Cihlar, J., 1996. Retrieving leaf area index of boreal conifer forests using Landsat Thematic Mapper, Remote Sening of Environment, 55, 153-162.

Coulibaly, L., and Goita, K., 2006. Evaluation of the potential of various spectral indices and textural features derived from satellite images for surficial deposits mapping, International Journal of Remote Sensing 27(20), 4567-4584.

Deering, D.W., Rouse, J.W., Haas, R.H., and Schell, J.A., 1975. Measuring Forage Production of Grazing Units from Landsat MSS Data, $10^{\text {th }}$ Internatonal Symposium on Remote Sensing of Environment 2, 1169-1178.

Fassnacht, K.S., Gower, S.T., Mackenzie, M.D., Nordheim, E.V., and Lillesand, T.M., 1997. Estimating the leaf area index of north central Wisconsin forest using Landsat Thematic Mapper. Remote Sening of Environment, 61, 229-245.

Faust, N.L., 1989. Image Enhancement. In Allen Kent and James G. Williams (eds), Encyclopedia of Computer Science and Technology, vol. 20, Supplement 5. Marcel Dekker Inc.

Fernandes, R.A., Miller, J.R., Chen, J.M., and Rubinstein, I.G., 2004. Evaluating image-based estimates of leaf area index in boreal conifer stands over a range of scales using highresolution CASI imagery, Remote Sensing of Environment 89, 200-216.

Goel, N.S., 1988. Models of vegetation canopy reflectance and their use in estimation of biophysical parameters from reflectance data, Remote Sens. Rev., 4, 1-212.

Goel, N.S., 1989. Inversion of canopy reflectance models for estimation of biophysical parameters from reflectance data. In G. Asrar (ed.), Theory and applications of optical remote sensing (205-251).

Haboudane, D., Miller, J.R., Pattey, E., Zarco-Tejada, P.J., Strachan, I.B., 2004. Hyperspectral vegetation indices and novel algorithms for predicting green LAI of crop canopies: 
Modeling and validation in the context of precision agriculture, Remote Sensing of Environment 90, 337-352.

Huete, A.R., 1988. A soil-adjusted vegetation index (SAVI), Remote Sensing of Environment 25, 295-309.

Jensen, R.J., 1996. Introductory Digital Image Processing: A Remote Sensing Perspective, Prentice Hall. 316pp.

Liang, S., 2004. Quantitative Remote Sensing, Wiley. 534pp.

Nemani, R., Pierce, L., Running, S., and Band, L., 1993. Forest ecosystem processes at the watershed scale: Sensitivity to remotely-sensed leaf area index estimates, International Journal of Remote Sensing, 14, 2519-2534.

Qi, J., Chehbouni, A., Huete, A.R., Kerr, Y.H., and Sorooshian, S., 1994. A modified soil adjusted vegetation index, Remote Sensing of Environment 48(2), 119-126.

Rahman, M.M., Csaplovics, E., and Koch, B., 2005. An efficient regression strategy for extracting forest biomass information from satellite sensor data, International Journal of Remote Sensing, 26(7), 1511-1519.

Rondeaux, G., Steven, M., and Baret, F., 1996. Optimization of soil-adjusted vegetation indices, Remote Sensing of Environment, 55, 95-107.

Rouse, J.W., Haas, R.H., Schell, J.A., and Deering, D.W., 1973. Monitoring vegetation systems in the Great Plains with ERTS, $3^{\text {rd }}$ ERTS Symposium, vol. 1, 48-62.

Skianis, G. Aim., Vaiopoulos, D., and Nikolakopoulos, K., 2004a. Assesment of the TVI vegetation index with the aid of probability theory, Proceedings of the $10^{\text {th }}$ International Congress of the Geological Society of Greece, Thessaloniki, vol. 3, 1338-1346.

Skianis, G. Aim., Vaiopoulos, D., and Nikolakopoulos, K., 2004b. A study of the behavior of vegetation index SAVI, based on probability theory, Proceedings of the $7^{\text {th }}$ Panhellenic Congress of the Hellenic Geographical Society, Mytilini, Greece, vol. 1, 41-48.

Spanner, M.A., Pierce, L.L., Peterson, D.L., and Running, S.W., 1990. Remote sensing of temperate coniferous forest leaf area index. The influence of canopy closure understory vegetation and background reflectance, Inernational Journal of Remote Sensing, 11, 95111.

Spiegel, M.R., 1977. Probability and Statistics, McGraw-Hill New York, ESPI Athens, 384pp.

Vaiopoulos, D., Skianis, G. Aim., and Nikolakopoulos, K., 2004. The contribution of probability theory in assessing the efficiency of two frequently used vegetation indices, International Journal of Remote Sensing 25(20), 4219-4236.

Verhoef, W., 1998. Theory of radiative transfer models applied in optical remote sensing of vegetation canopies, Wageningen: Grafish Service Centrum Van Gils. 